\title{
The impact of enhanced recovery after surgery (ERAS) protocol compliance on morbidity from resection for primary lung cancer
}

\author{
Luke J. Rogers, BSc, MBChB, MRCS, ${ }^{a}$ David Bleetman, BA, MB BS, MRCS, \\ David E. Messenger, BMedSc, MBChB, FRCS, ${ }^{\mathrm{c}}$ Natasha A. Joshi, MBChB, MRCP, FRCA, ${ }^{\mathrm{d}}$ Lesley Wood, ${ }^{\mathrm{e}}$ \\ Neil J. Rasburn, MBChB, FRCA, ${ }^{d}$ and Timothy J. P. Batchelor, BSc, MBChB, MSc, FRCS (CTh)
}

\section{ABSTRACT}

Objective: The adoption of Enhanced Recovery After Surgery programs in thoracic surgery is relatively recent with limited outcome data. This study aimed to determine the impact of an Enhanced Recovery After Surgery pathway on morbidity and length of stay in patients undergoing lung resection for primary lung cancer.

Methods: This prospective cohort study collected data on consecutive patients undergoing lung resection for primary lung cancer between April 2012 and June 2014 at a regional referral center in the United Kingdom. All patients followed a standardized, 15-element Enhanced Recovery After Surgery protocol. Key data fields included protocol compliance with individual elements, pathophysiology, and operative factors. Thirty-day morbidity was taken as the primary outcome measure and classified a priori according to the Clavien-Dindo system. Logistic regression models were devised to identify independent risk factors for morbidity and length of stay.

Results: A total of 422 consecutive patients underwent lung resection over a 2 year period, of whom 302 (71.6\%) underwent video-assisted thoracoscopic surgery. Lobectomy was performed in 297 patients $(70.4 \%)$. Complications were experienced by 159 patients $(37.6 \%)$. The median length of stay was 5 days (range, 1-67), and 6 patients (1.4\%) died within 30 days of surgery. There was a significant inverse relationship between protocol compliance and morbidity after adjustment for confounding factors (odds ratio, $0.72 ; 95 \%$ confidence interval, $0.57-0.91 ; P<.01)$. Age, lobectomy or pneumonectomy, more than 1 resection, and delayed mobilization were independent predictors of morbidity. Age, lack of preoperative carbohydrate drinks, planned high dependency unit/intensive therapy unit admission, delayed mobilization, and open approach were independent predictors of delayed discharge (length of stay $>5$ days).

Conclusions: Increased compliance with an Enhanced Recovery After Surgery pathway is associated with improved clinical outcomes after resection for primary lung cancer. Several elements, including early mobilization, appear to be more influential than others. (J Thorac Cardiovasc Surg 2018;155:1843-52)

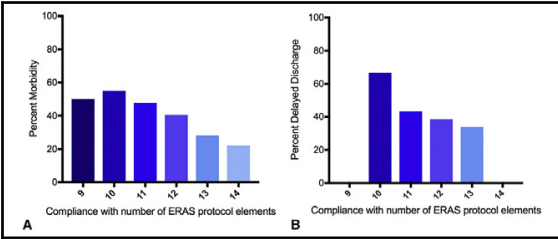

Compliance with number of ERAS protocols and (A) morbidity and (B) delayed discharge.

Central Message

Increasing overall compliance with an ERAS pathway in patients undergoing lung cancer resection is associated with a reduction in postoperative morbidity and a shorter LOS.

\section{Perspective}

ERAS protocols have been described in thoracic surgery, but there remains a paucity of evidence reporting clinical outcomes. In this study, we have demonstrated that increased overall compliance with ERAS protocols reduces morbidity and LOS. In addition, several independent factors that seem more influential than other elements of the pathway have been identified.

See Editorial Commentary page 1853

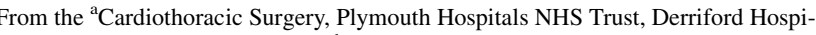
tal, Plymouth, United Kingdom; ${ }^{\mathrm{b}}$ Cardiothoracic Surgery, Barts Health NHS Trust, Barts Heart Centre, St Bartholomews Hospital, London, United Kingdom; ${ }^{\text {CColor- }}$ ectal Surgery, ${ }^{\mathrm{d}}$ Anesthesia, ${ }^{\mathrm{e}}$ Database Manager, and ${ }^{\mathrm{f}}$ Thoracic Surgery, University Hospitals Bristol NHS Foundation Trust, Bristol Royal Infirmary, Bristol, United Kingdom.

Above \& Beyond Charity at University Hospitals Bristol NHS Foundation Trust provided grants to present this work at the Boston American Association for Thoracic Surgery following its completion.
}

Read at the 97th Annual Meeting of The American Association for Thoracic Surgery, Boston, Massachusetts, April 29-May 3, 2017.

Received for publication May 4, 2017; revisions received Oct 12, 2017; accepted for publication Oct 24, 2017; available ahead of print Jan 17, 2018

Address for reprints: Luke J. Rogers, BSc, MBChB, MRCS, Southwest ST2 Cardiothoracic Surgery, Cardiothoracic Surgery, Plymouth Hospitals NHS Trust, Derriford Rd, Plymouth, PL6 8DH (E-mail: ljrogers@doctors.org.uk). 0022-5223/\$36.00

Copyright (c) 2017 by The American Association for Thoracic Surgery https://doi.org/10.1016/j.jtcvs.2017.10.151 


\section{Abbreviations and Acronyms}

ERAS $=$ Enhanced Recovery After Surgery

LOS = length of stay

$\mathrm{OR}=$ odds ratio

$\mathrm{PONV}=$ postoperative nausea and vomiting

VATS $=$ video-assisted thoracoscopic surgery

Scanning this $Q R$ code will take

you to a supplemental video. To view the AATS Annual Meeting Webcast, see the URL next to the webcast thumbnail.

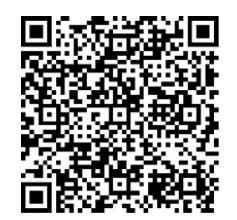

Lung cancer is the leading cause of cancer deaths worldwide, accounting for $13 \%$ and $14 \%$ of all new cancers in the United Kingdom and United States, respectively. ${ }^{1,2}$ Surgical resection offers the best chance of cure for earlystage lung cancer and therefore represents the first choice treatment. ${ }^{3}$ However, lung cancer is associated with significant complications in up to $50 \%$ of cases, which can lead to delayed recovery, poorer long-term outcomes, and higher costs. ${ }^{4,5}$

Enhanced Recovery After Surgery (ERAS) pathways were initially developed in colorectal surgery in an effort to improve the postoperative recovery of patients undergoing colonic resection, based largely on the work of Kehlet and others. ${ }^{6-9}$ ERAS pathways consist of multimodal, evidence-based protocols targeting the entire patient pathway from referral to discharge. The stress response to surgery is reduced and results in improved patient outcomes. ${ }^{8}$ Furthermore, variations in treatment are reduced, earlier hospital discharge is facilitated, and hospital costs are lowered. ${ }^{6,10-12}$

Fast-track, multimodal pathways have been described in thoracic surgery. Cerfolio and colleagues ${ }^{13}$ demonstrated that preoperative patient education and standardized removal of catheters and chest drains postoperatively facilitated earlier discharge without adversely affecting morbidity or mortality. Other groups have demonstrated a reduction in complications and a reduced length of stay (LOS). ${ }^{14-17}$ More recently, specific ERAS pathways for thoracic surgery have been described. ${ }^{18-20}$ Nevertheless, there remains a paucity of evidence regarding ERAS in lung resection surgery, particularly in terms of clinical outcomes and in combination with a predominantly minimally invasive video-assisted thoracoscopic surgery (VATS) technique.

It remains unclear whether the benefits obtained from ERAS pathways are a consequence of a significant improvement in 1 or 2 individual elements of care or whether they are the aggregation of marginal gains throughout the pathway. In this study, we present findings from a large institutional database of an ERAS program that includes all patients undergoing primary lung resection during the study period. Our primary aim was to identify the individual elements of an ERAS protocol predictive of 30day morbidity and delayed discharge in patients undergoing resection for lung cancer.

\section{MATERIAL AND METHODS}

This study was reported according to the STROBE criteria for observational studies. ${ }^{21}$ Institutional approval was obtained for the conduct of the study as an audit of practice.

\section{Patient Selection}

In this cohort study, patients undergoing lung resection for primary lung cancer at a single UK regional referral center between April 2012 and June 2014 were prospectively identified and entered into an electronic database. All adult patients (aged $>18$ years) were included if they underwent a sublobar resection (anatomic segmentectomy or nonanatomic wedge resection), lobectomy, or pneumonectomy for primary lung cancer regardless of surgical approach. Elective and expedited operations were included. Those undergoing thoracic surgical operations for benign disease or metastatic disease, and individuals aged less than 18 years were excluded.

Key data fields collected included patient demographics, pathophysiology, protocol compliance with individual elements of an ERAS protocol, and operative factors. The primary outcome measure was any 30-day morbidity, which was classified a priori according to the Clavien-Dindo system specific to thoracic surgery. Minor morbidity was defined as the occurrence of a Clavien-Dindo grade I or II complication, and major morbidity was defined as the occurrence of a grade III or IV complication. Postoperative LOS was considered a secondary outcome measure and dichotomized around the median LOS and thus treated as a categoric variable. Other secondary outcome measures included 30-day readmission, reoperation, and 30-day mortality.

\section{Enhanced Recovery After Surgery}

ERAS protocols were established in 2010 to improve patient outcomes, reduce variation in practice, and provide more cost-effective care. All patients followed the institution's generic, standardized 15-element ERAS protocol, against which protocol compliance was determined (Table 1 and Video 1). Patients were reviewed daily, including weekends, by an attending thoracic surgeon.

The standard multimodal approach can be divided into preoperative, perioperative, and postoperative phases. Further elements specific to lung resection surgery were included to form our institution's thoracic surgery ERAS pathway, as detailed next and in Table 2.

\section{Preoperative Phase}

All patients attended a multidisciplinary 1-stop clinic for preoperative assessment. They were assessed by a surgeon, an anesthesiologist, and a nurse skilled in the preoperative preparation of patients. This enabled the early identification and management of comorbidities and facilitated subsequent day-of-surgery admission. Patients received an ERAS diary that outlined the protocol, provided daily targets and milestones, and introduced the concept of a planned date of discharge after elective surgery. The diary was then completed during their admission. Smokingcessation counseling was provided when necessary, but smoking status did not delay admission. If patients were deemed unfit for surgery, they 
TABLE 1. Elements of the generic Enhanced Recovery After Surgery pathway

1. Preoperative visit

2. Preoperative assessment

3. Patient education and explanation of ERAS

4. Admission on day of surgery

5. Preoperative carbohydrate drink

6. Avoidance of sedation

7. Prophylactic antibiotics

8. Regional anesthesia

9. Goal-directed fluid therapy

10. Intraoperative warming

11. Avoidance of postoperative intravenous fluids

12. Avoidance of opiate analgesia

13. Early feeding

14. Targeted PONV therapy

15. Mobilization within $24 \mathrm{~h}$

ERAS, Enhanced Recovery After Surgery; $P O N V$, postoperative nausea and vomiting.

were enrolled in a preoperative rehabilitation program consisting of $4 \mathrm{el}-$ ements: patient education, smoking cessation, nutritional advice, and exercise (based on chronic obstructive pulmonary disease rehabilitation programs).

All patients were admitted to hospital on the day of their surgery. Two hours before induction of anesthesia, patients were given complex carbohydrate drinks to prevent dehydration, reduce postoperative insulin resistance, and improve preoperative well-being. ${ }^{22}$ Routine administration of preanesthetic sedative medication was not given, and patients walked from the preoperative department to the operating room.

\section{Perioperative Phase}

Prophylactic antibiotics were given at induction. General anesthesia with lung-protective strategies during 1-lung ventilation was used in combination with regional anesthesia (normally a paravertebral catheter with additional intercostal blocks). Epidural anesthesia was not routinely used. Balanced crystalloid solutions were infused intravenously, and vasopressors were administered to avoid intraoperative hypoperfusion. Goal-

University Hospitals Bristol WHS NHS Foundation Trust

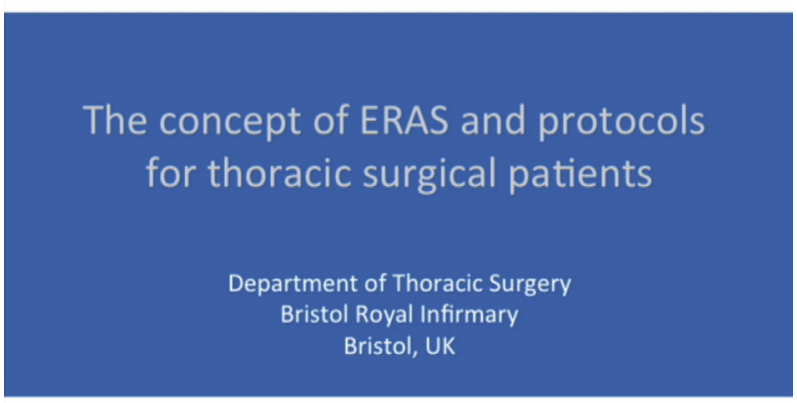

VIDEO 1. The concept of ERAS and protocols for patients undergoing thoracic surgery. Video available at: http://www.jtcvsonline.org/article/ S0022-5223(17)32855-6/fulltext.
TABLE 2. Thoracic surgery Enhanced Recovery After Surgery pathway

\begin{tabular}{l} 
Preoperative phase \\
1. Preoperative visit and assessment \\
2. Patient education and explanation of ERAS \\
3. Smoking cessation \\
4. Preoperative rehabilitation \\
5. Admission on day of surgery \\
6. Preoperative carbohydrate drink \\
7. Avoidance of sedative preanesthetic medication \\
Perioperative phase \\
1. Prophylactic antibiotics \\
2. Regional anesthesia with paravertebral catheters \\
3. Avoidance of crystalloid overload \\
4. Intraoperative warming \\
5. Venous thromboembolism prophylaxis \\
6. Avoidance of urinary catheter \\
7. Minimally invasive surgery (VATS) where possible \\
8. Single chest drain \\
Postoperative phase \\
1. Avoidance of postoperative intravenous fluids \\
2. Avoidance of opiate analgesia \\
3. Early feeding \\
4. Targeted PONV therapy \\
5. Mobilization within 24 h \\
6. Early chest drain and catheter removal \\
\hline
\end{tabular}

ERAS, Enhanced Recovery After Surgery; VATS, video-assisted thoracoscopic surgery; $P O N V$, postoperative nausea and vomiting.

directed fluid therapy and the use of noninvasive cardiac output monitors were not found to be useful. Instead, anesthesiologists avoided restrictive or liberal fluid regimens.

Ketamine and intravenous steroids were used as adjuncts to pain relief and postoperative nausea and vomiting (PONV). Normothermia was maintained throughout using convective active warming devices. Venous thromboembolism prophylaxis consisted of compression stockings and pneumatic compression devices intraoperatively, with chemoprophylaxis in the form of subcutaneous low-molecular-weight heparin started on the evening of surgery. Urinary catheters were not routinely placed.

Minimally invasive (VATS) surgery was used wherever possible. A single chest drain was placed at the end of each procedure and connected to a Thopaz digital chest drainage system (Medela, Switzerland).

\section{Postoperative Phase}

Intravenous fluids were replaced by oral fluids and diet at the earliest opportunity, typically by the morning after surgery. A standardized analgesic regimen was used consisting of regular acetaminophen, and nonsteroidal anti-inflammatory drugs where appropriate. Opiates were avoided if possible and used only for breakthrough pain if required. Paravertebral blockade continued until postoperative day 2. PONV was addressed with a multimodal approach consisting of both nonpharmacologic (preoperative carbohydrate drinks, avoidance of crystalloid overload) and pharmacologic (avoidance of opiates, steroids, and regular postoperative ondansetron) components.

Patients were mobilized within 24 hours of surgery and had milestones to achieve each day. Thoracic surgery physiotherapists provided daily sessions with all patients to improve ventilation, aid clearance of secretions, and encourage mobilization. 
Chest drains were removed early according to permissive protocols. This required the absence of an air leak for the previous 6 hours and the drainage of less than $500 \mathrm{~mL}$ fluid for the previous 24 hours.

\section{Statistical Methods}

Continuous variables are expressed as median and interquartile range, and normality was assessed with the Shapiro-Wilk test. Categoric variables are expressed as count and percentages. Patients were divided in 2 groups according to the presence of any morbidity event at 30 days from surgery; 159 patients had such an event, and the remaining 263 patients did not.

The analysis between groups was conducted using the Mann-Whitney $U$ test for continuous variables that were not normally distributed. Categoric variables were compared with Pearson's chi-square test or Fisher exact test as appropriate. Predictors for morbidity at 30 days, as defined by a $P$ value less than .10 on univariate analysis, were tested using a parsimonious multiple logistic regression model. A stepwise approach was conducted by backward and forward selection methods using Akaike information criterion as discrimination criterion between models. The predictive ability of the model was tested with the Hosmer-Lemeshow goodness of fit test. The same method of analysis was used to define the independent predictors of prolonged postoperative LOS. This was defined as a LOS equal to or greater than 5 postoperative days. Alpha error was set at 0.05 , and all tests were 2 tailed. Statistical analysis was performed with $R$ version 3.4.0 (R Foundation for Statistical Computing, Vienna, Austria).

\section{RESULTS}

A total of 422 patients underwent lung resection for primary lung cancer between April 2012 and June 2014. A total of $302(71.6 \%)$ of these resections were performed using a VATS approach. Forty-one $(9.7 \%)$ VATS procedures were converted to open, and $77(18.2 \%)$ were performed as a planned open procedure. Elective procedures accounted for 416 cases $(98.6 \%)$, and the remaining cases were made up of expedited cases brought forward from their originally planned day of surgery because of clinical deterioration. Multiple resections were performed in 47 patients $(11.1 \%)$. Lobectomy was the most frequently performed procedure and accounted for 297 (70.4\%) of cases. Sublobar resections and pneumonectomies accounted for 111 cases $(26.3 \%)$ and 14 cases $(3.3 \%)$, respectively.

A total of 154 patients $(36.5 \%)$ had a Thoracoscore of 2 or greater, and 118 patients $(28.0 \%)$ had an American Society of Anesthesiologists of III or greater. A total of 356 patients $(84.4 \%)$ had a World Health Organization performance status of 0 or 1 , with 343 patients $(81.3 \%)$ having a Medical Research Council dyspnea score of 0 or 1. There were $7(1.6 \%)$ unplanned reoperations and 23 $(5.5 \%)$ readmissions within 30 days of the primary operation (Table 3). Complications were experienced by 159 patients $(37.6 \%)$. Of these, $55(13 \%)$ were major complications (Clavien-Dindo III and IV).

\section{Predictors of Morbidity}

Multiple logistic regression identified sublobar resection (odds ratio [OR], 0.45; 95\% confidence interval [CI], 0.27$0.75 ; P<.01)$ as an independent predictor of reduced morbidity at 30 days (Table 4 ), whereas increased age
TABLE 3. Patient outcomes

\begin{tabular}{lc}
\hline LOS, median (range) & $5(1-67)$ \\
\hline HDU LOS, median (range) & $0(0-10)[2(1-10)]^{* \dagger}$ \\
\hline ITU LOS, median (range) & $0(0-36)[3(1-36)]^{* \dagger}$ \\
30-d readmission, n (\%) & $23(5.5)$ \\
30-d reoperation, n (\%) & $7(1.6)$ \\
30-d morbidity, n (\%) & \\
$\quad$ None & $259(61.4)$ \\
Minor (Clavien-Dindo I and II) & $104(24.6)$ \\
Major (Clavien-Dindo III and IV) & $55(13.0)$ \\
Missing & $4(0.9)$ \\
Mortality, n (\%) & \\
$30 \mathrm{~d}$ & $6(1.4)$ \\
$60 \mathrm{~d}$ & $7(1.7)$ \\
$90 \mathrm{~d}$ & $9(2.1)$ \\
\hline
\end{tabular}

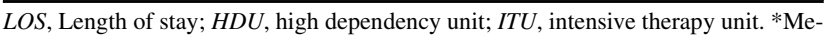
dian and range for the entire population. $\dagger$ Median and range for patients admitted in HDU. ‡Media and range for patients admitted to the ITU.

(OR, 1.02 per year; 95\% CI, 1.00-1.02, $P=.04$ ) and more than 1 resection (OR, 2.21; 95\% CI, 1.13-4.36; $P=.02)$ were predictive of increased morbidity. There was a significant inverse relationship between protocol compliance and morbidity (OR, 0.72; 95\% CI, 0.57-0.91; $P<.01$ ) (Figure 1, A).

\section{Predictors of Length of Stay}

Planned admission to the high dependency unit (OR, 2.86; 95\% CI, 1.64-5.03; $P<.01)$ or intensive therapy unit (OR, 3.81; 95\% CI, 1.82-8.61; $P<.01)$ was an independent predictor of delayed discharge (LOS $>5$ days), whereas surgery via a VATS approach (OR, 0.54; $95 \%$ CI, 0.32-0.89; $P=.01$ ), preoperative carbohydrate drinks (OR, $0.57 ; 95 \%$ CI, 0.36-0.96; $P=.01$ ), and early mobilization (OR, 0.25 ; $95 \% \mathrm{CI}, 0.16-0.40 ; P<.01)$ were associated with reduced LOS (Table 5).

\section{DISCUSSION}

In this study, it has been shown that overall compliance with an ERAS pathway is important for patients undergoing lung resection for primary lung cancer. Increased compliance was associated with reduced morbidity. At the same time, several independent factors emerged as being more influential than other elements of the pathway. Early mobilization and preoperative carbohydrate drinks were the only individual protocol elements that were predictive of reduced morbidity or LOS. Use of the VATS approach was associated with a reduction in LOS, but not morbidity. Other factors associated with increased morbidity were more than 1 resection, lobectomy or pneumonectomy, and age. Planned admission to the high dependency unit/intensive therapy unit was an important institutional factor associated with delayed discharge, although it had no impact on 
TABLE 4. Predictors of morbidity at 30 days

\begin{tabular}{|c|c|c|c|c|c|c|}
\hline & \multicolumn{3}{|c|}{ Univariable analysis } & \multicolumn{3}{|c|}{ Multivariable analysis } \\
\hline & $\overline{\mathbf{O R}}$ & $95 \% \mathrm{CI}$ & $P$ value & $\overline{\mathbf{O R}}$ & $\mathbf{9 5} \% \mathrm{CI}$ & $P$ value \\
\hline Age & 1.03 & $1.01-1.05$ & .01 & 1.02 & $1.001-1.05$ & .04 \\
\hline Gender (male) & 0.66 & $0.44-0.98$ & .04 & & & \\
\hline Sublobar resection & 0.54 & $0.33-0.86$ & .01 & 0.45 & $0.27-0.75$ & $<.01$ \\
\hline$>1$ resection & 2.04 & $1.11-3.79$ & .02 & 2.21 & $1.13-4.36$ & .02 \\
\hline VATS procedure & 0.96 & $0.62-1.49$ & .87 & & & \\
\hline Thoracoscore & 0.97 & 0.86-1.09 & .68 & & & \\
\hline Preoperative visit & NA & NA & NA & & & \\
\hline Preoperative assessment & NA & NA & NA & & & \\
\hline Explanation ERAS & 1.28 & $0.65-2.64$ & .48 & & & \\
\hline Day of surgery admission & 0.30 & $0.04-1.54$ & .16 & & & \\
\hline Carbohydrate drink & 0.62 & $0.41-0.98$ & .04 & & & \\
\hline Avoidance of sedation & NA & NA & NA & & & \\
\hline Prophylactic antibiotic & 0.52 & $0.19-1.4$ & .19 & & & \\
\hline Regional anesthesia & 0.74 & $0.34-1.66$ & .45 & & & \\
\hline Intraoperative warming & NA & NA & NA & & & \\
\hline Avoidance of IV fluid & 0.30 & $0.01-3.15$ & .33 & & & \\
\hline Avoidance of opiate analgesia & 1.34 & $0.38-3.72$ & .61 & & & \\
\hline Early feeding & 0.49 & $0.22-1.11$ & .08 & & & \\
\hline Targeted PONV treatment & 1.32 & $0.57-3.32$ & .52 & & & \\
\hline Early mobilization & 0.47 & $0.31-0.72$ & $<.01$ & & & \\
\hline Preoperative creatinine & 1.01 & $1.002-1.2$ & .02 & 1.01 & $0.99-1.01$ & .07 \\
\hline Preoperative hemoglobin & 1.01 & $0.99-1.02$ & .09 & & & \\
\hline Compliance score & 0.68 & $0.54-0.84$ & $<.01$ & 0.72 & $0.57-0.91$ & $<.01$ \\
\hline HDU stay & 4.95 & $3.03-8.36$ & $<.01$ & & & \\
\hline ITU stay & 6.51 & $3.35-14$ & $<.01$ & 2.00 & $1.18-3.6$ & .02 \\
\hline
\end{tabular}

$O R$, Odds ratio; $C I$, confidence interval; VATS, video-assisted thoracic surgery; $N A$, not available; $E R A S$, Enhanced Recovery After Surgery; $I V$, intravenous; PONV, postoperative nausea and vomiting; $H D U$, high dependency unit; $I T U$, intensive care unit.

morbidity. Earlier work by Madani and colleagues ${ }^{18}$ also noted that the whole pathway was likely to be more important than individual elements, but did demonstrate that early chest drain and urinary catheter removal were independent predictors of a shorter hospital stay.

ERAS describes multimodal protocols designed to optimize patients preoperatively, reduce and limit the stress response to surgery perioperatively, and improve postoperative recovery, facilitating a more timely return to a patient's normal activities of daily living. Sometimes referred to as "Enhanced Recovery Programs" or "fasttrack surgery," these pathways have revolutionized the care of surgical patients across a number of surgical specialties since their introduction in gastrointestinal surgery in the late 1990s. ${ }^{6}$ Patient outcomes are optimized, morbidity is reduced, and variations in care are minimized. These efficiencies then translate into a shorter length in hospital stay and reduced costs without affecting readmission rates. ${ }^{10-12}$ There is little, strong level 1 evidence to suggest ERAS pathways are superior to conventional care in thoracic surgery, although this study appears to demonstrate that increased compliance with the protocol improves clinical outcomes.

The adoption of the ERAS concept in thoracic surgery is relatively recent. Evidence for the benefit of ERAS protocols is limited, and few, if any, have addressed the impact of VATS on outcome within their program. Cerfolio and colleagues ${ }^{13}$ implemented a fast-track program for open lung resections with a particular focus on preoperative patient education, epidural use, standardized aggressive removal of catheters and chest drains postoperatively, early mobilization, and a daily plan with an estimated day of discharge on the fourth postoperative day. These interventions facilitated early discharge without adversely affecting morbidity or mortality. ${ }^{13}$ Other groups have demonstrated a reduction in complications and a reduced LOS using fasttrack protocols. ${ }^{14-17}$ In one small, randomized controlled trial, a pathway involving the avoidance of preoperative fasting, regional analgesia, early enteral feeding, and early ambulation resulted in a significant reduction in 


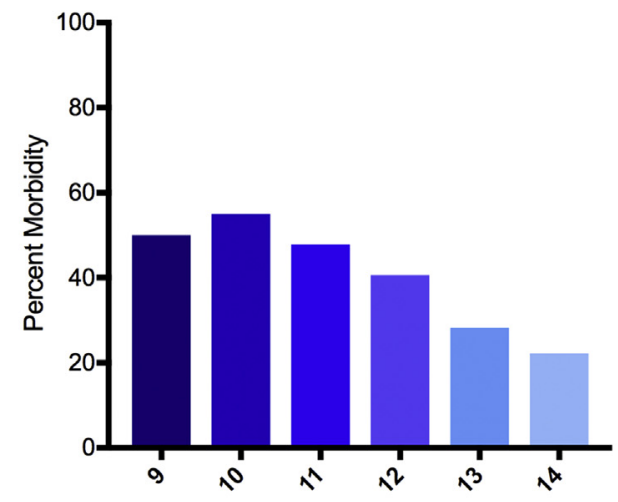

A

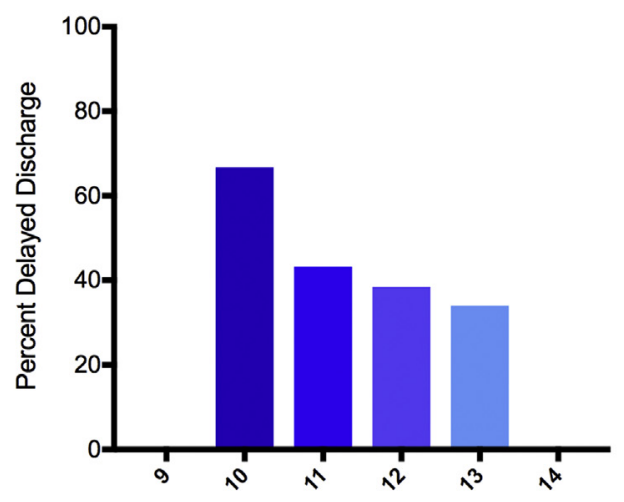

B

FIGURE 1. Association between compliance with number of ERAS protocol elements and (A) morbidity and (B) delayed discharge. ERAS, Enhanced Recovery After Surgery.

pulmonary complications. ${ }^{16}$ With the use of propensity matching, Salati and colleagues ${ }^{14}$ were able to show that the introduction of a fast-track pathway resulted in a reduced LOS. Their protocols focused on preoperative patient education, standardizing postoperative care, and aggressive chest drain management.

TABLE 5. Predictors of prolonged length of stay

\begin{tabular}{|c|c|c|c|c|c|c|}
\hline & \multicolumn{3}{|c|}{ Univariable analysis } & \multicolumn{3}{|c|}{ Multivariable analysis } \\
\hline & $\overline{\mathbf{O R}}$ & $95 \% \mathrm{CI}$ & $\overline{P \text { value }}$ & OR & $95 \% \mathrm{CI}$ & $P$ value \\
\hline Age & 1.03 & $1.01-1.05$ & $<.01$ & 1.03 & $1.01-1.06$ & $<.01$ \\
\hline Gender (male) & 0.78 & $0.53-1.15$ & .21 & & & \\
\hline Sublobar resection & 0.66 & $0.43-1.03$ & .07 & & & \\
\hline$>1$ resection & 1.23 & $0.67-2.29$ & .50 & & & \\
\hline VATS procedure & 0.39 & $0.25-0.61$ & $<.01$ & 0.54 & $0.32-0.89$ & .01 \\
\hline Thoracoscore & 1.05 & $0.94-1.18$ & .38 & & & \\
\hline Preoperative visit & NA & NA & NA & & & \\
\hline Preoperative assessment & NA & NA & NA & & & \\
\hline Explanation ERAS & 1.16 & $0.84-3.19$ & .16 & & & \\
\hline Day of surgery admission & 0.20 & $0.01-1.27$ & .15 & & & \\
\hline Carbohydrate drink & 0.57 & $0.36-0.89$ & .01 & 0.59 & $0.36-0.96$ & .04 \\
\hline Avoidance of sedation & NA & NA & NA & & & \\
\hline Prophylactic antibiotic & 0.54 & $0.19-1.47$ & .25 & & & \\
\hline Regional anesthesia & 2.16 & $0.97-5.14$ & .07 & & & \\
\hline Intraoperative warming & NA & NA & NA & & & \\
\hline Avoidance of IV fluid & NA & NA & NA & & & \\
\hline Avoidance of opiate analgesia & 1.05 & $0.47-2.39$ & .90 & & & \\
\hline Early feeding & 0.74 & $0.33-1.65$ & .47 & & & \\
\hline Targeted PONV treatment & 1.59 & $0.70-3.73$ & .27 & & & \\
\hline Early mobilization & 0.25 & $0.16-0.40$ & $<.01$ & 0.43 & $0.26-0.71$ & $<.01$ \\
\hline Preoperative creatinine & 1.01 & $1.001-1.02$ & .04 & & & \\
\hline Preoperative hemoglobin & 0.99 & $0.98-1.01$ & .38 & & & \\
\hline Compliance score & 0.67 & $0.53-0.83$ & $<.01$ & & & \\
\hline HDU admission & 4.95 & $3.02-8.36$ & $<.01$ & 2.86 & $1.64-5.03$ & $<.01$ \\
\hline ITU admission & 6.51 & $3.35-14$ & $<.01$ & 3.81 & $1.82-8.61$ & $<.01$ \\
\hline
\end{tabular}

$O R$, Odds ratio; $C I$, confidence interval; VATS, video-assisted thoracic surgery; $N A$, not available; $E R A S$, enhanced recovery after surgery; $I V$, intravenous; $P O N V$, postoperative nausea and vomiting; $H D U$, high dependency unit; $I T U$, intensive therapy unit. 
More recently, specific ERAS pathways for thoracic surgery have been described. ${ }^{18-20}$ Madani and colleagues ${ }^{18}$ introduced an ERAS program for open lobectomy, standardizing care and addressing preoperative, intraoperative, and postoperative elements of the patient pathway. They were able to demonstrate a reduction in LOS and a reduced number of complications without an increase in readmission rates. However, their protocols were relatively conservative. A move away from epidural analgesia to paravertebral blockade and more aggressive chest drain protocols may have allowed them to demonstrate even greater benefits. $^{23-26}$

A number of different elements make up ERAS programs. It has not been possible to say whether some elements are more influential than others or whether adherence to the pathway in its entirety is more important. Experience in colorectal cancer surgery indicates that both options may hold true. ${ }^{27}$ Increasing adherence to an ERAS pathway was associated with improved clinical outcomes, specifically reduced morbidity, symptoms, and readmissions. At the same time, restriction of intravenous fluids and the use of preoperative carbohydrate drinks were independent predictors of improved outcomes. Results from a large international registry on ERAS compliance in colorectal cancer surgery have corroborated these earlier results, while also demonstrating a reducing LOS with increasing compliance. ${ }^{28}$ Furthermore, minimally invasive surgery was identified as an independent factor. Other independent factors for a shorter hospital stay included preoperative carbohydrate loading, total intravenous anesthesia, and avoidance of epidural analgesia, whereas restriction of perioperative intravenous fluids was associated with fewer complications.

The feared consequence of introducing a fast-track program is that readmissions will increase. A readmission is harmful to patients with lung cancer and is associated with reduced short-term and long-term survival. ${ }^{29}$ These concerns have not been realized in colorectal cancer surgery or other specialties. ${ }^{10,11}$ Likewise, an increase in readmissions has not been demonstrated after lung cancer surgery within a fast-track or ERAS program. ${ }^{14,18}$ In this study, our 30-day readmission rate was $5.5 \%$. This is in keeping with other studies and registries. ${ }^{14,29}$ However, one shortfall of this study is the inability to determine whether our ERAS pathway had an adverse effect on readmissions, because we have not included a comparator group before the introduction of the program.

Minimally invasive surgery appears to be an independent predictor of a favorable outcome after colorectal cancer surgery within ERAS programs. ${ }^{28,30}$ VATS lung resection is increasing in popularity as evidence emerges of its efficacy in treating lung cancer while potentially improving outcomes. ${ }^{31,32}$ This study is the first to look at a well-established VATS program within an ERAS pathway.
VATS lung resection was an independent predictor of LOS but not a predictor of morbidity.

Immobility after thoracic surgery is common and largely due to modifiable factors, such as pain, nausea, drowsiness, continued chest drainage, and feeling light headed.$^{33}$ In this study, we have demonstrated that mobilization within the first 24 hours is beneficial in terms of LOS and morbidity. Early mobilization has been identified as a factor in reducing complications in a fast-track program, ${ }^{17}$ although a recent systematic review did not identify any benefits because of the poor quality of studies and conflicting results. ${ }^{34}$ Several elements of our ERAS pathway are likely to positively influence early mobilization. These include targeted PONV control, avoidance of epidural analgesia, a standardized analgesia regimen with avoidance of opiates where possible, and avoidance of fluid overload. The use of a digital chest drainage system with aggressive chest drain management may help. ${ }^{35}$

\section{Study Limitations}

A limitation of this study is the lack of a control group. This has been recognized as a potential problem in studies looking at the effects of ERAS and fast-track pathways in thoracic surgery. ${ }^{36}$ Indeed, there has only been 1 small and underpowered randomized controlled trial reported. ${ }^{16}$ Consequently, making statements about the ability of an ERAS pathway to reduce morbidity and shorten hospital stay when compared with traditional care is likely to introduce a number of biases, including selection, detection, and performance bias. ${ }^{36}$ In this study, we made no comparison with traditional care. All patients in our institution are on an ERAS pathway irrespective of procedure, comorbidities, or surgical approach, and this article reports consecutive patients admitted under our care over a 2-year period. A randomized controlled trial would be ideal but is likely to prove impossible to run because there would be considerable contamination between control and study groups managed in the same institution on the same ward by the same team. Another limitation of our study is the potential risk for postoperative ERAS compliance to be affected by the presence of early complications. However, in analysis of LOS in which morbidity was excluded and therefore the potential for reverse causality limited, a relationship of compliance with ERAS protocols remained even when including those patients admitted to the intensive therapy unit/high dependency unit (and therefore most likely to develop an early complication).

\section{CONCLUSIONS}

In patients undergoing lung cancer resection within an ERAS program, increased compliance with ERAS protocols is associated with a reduction in postoperative morbidity. Early mobilization after surgery is independently associated with reduced morbidity and LOS. In 
addition, VATS lung resection is associated with reduced LOS. Although it is clear that greater adherence to an ERAS pathway is associated with improved clinical outcomes, several elements appear to be more influential than others. ERAS pathways should be considered for care of all patients undergoing surgery for primary lung cancer.

\section{Webcast}

You can watch a Webcast of this AATS meeting presentation by going to: https://aats.blob.core.windows.net/media/ 17AM/2017-05-02/RM302-304/05-02-17_Room302-304_ 1400_Rogers.mp4.

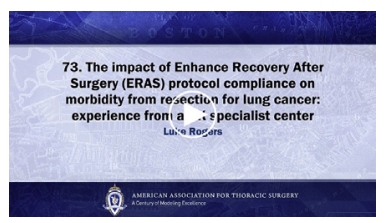

\section{Conflict of Interest Statement}

Authors have nothing to disclose with regard to commercial support.

The authors thank V. D. Bruno of Wissenroad Ltd, for conducting the statistical review of the article.

\section{References}

1. Cancer Research UK. Lung cancer mortality statistics. 2014. Available at: http:// www.cancerresearchuk.org/health-professional/cancer-statistics/statistics-by-cancertype/lung-cancer\#heading-Zero. Accessed January 1, 2018.

2. American Cancer Society. Lung and bronchus cancer at a glance. 2017. Available at: https://cancerstatisticscenter.cancer.org/\#!/. Accessed January 1, 2018.

3. Royal College of Physicians. Lung Cancer Clinical Outcomes Publication 2016 (for the 2014 Audit Period). London: RCP; 2016.

4. Chen FF, Zhang D, Wang YL, Xiong B. Video-assisted thoracoscopic surgery lobectomy versus open lobectomy in patients with clinical stage non-small cell lung cancer: a meta-analysis. Eur J Surg Oncol. 2013;39:957-63.

5. Phillips JD, Merkow RP, Sherman KL, DeCamp MM, Bentrem DJ, Bilimoria KY. Factors affecting selection of operative approach and subsequent short-term outcomes after anatomic resection for lung cancer. J Am Coll Surg. 2012;215:206-15.

6. Kehlet H. Multimodal approach to control postoperative pathophysiology and rehabilitation. Br J Anaesth. 1997;78:606-17.

7. Wilmore DW, Kehlet H. Management of patients in fast track surgery. BMJ. 2001;322:473-6.

8. Kehlet H, Wimore DW. Multimodal strategies to improve surgical outcome. Am J Surg. 2002;183:630-41.

9. Fearon KCH, Lljungqvist O, Von Meyenfeldt M, Revhaug A, Dejong CHC Lassen K, et al. Enhanced recovery after surgery: a consensus review of clinical care for patients undergoing colonic resection. Clin Nutr. 2005;24:466-77.

10. Nicholson A, Lowe MC, Parker J, Lewis SR, Alderson P, Smith AF. Systematic review and meta-analysis of enhanced recovery programmes in surgical patients. Br J Surg. 2014;101:172-88.

11. Paton F, Chambers D, Wilson P, Eastwood A, Craig D, Fox D, et al. Effectiveness and implementation of enhanced recovery after surgery programmes: a rapid evidence synthesis. BMJ Open. 2014;4:e005015.

12. Lee L, Mata J, Ghitulescu GA, Boutros M, Charlebois P, Stein B, et al. Costeffectiveness of enhanced recovery versus conventional perioperative management for colorectal surgery. Ann Surg. 2015;262:1026-33.

13. Cerfolio RJ, Pickens A, Bass C, Katholi C. Fast-tracking pulmonary resections. J Thorac Cardiovasc Surg. 2001;122:318-24.
14. Salati M, Brunelli A, Xiume F, Refai M, Pompili C, Sabbatini A. Does fasttracking increase the readmission rate after pulmonary resection? A casematched study. Eur J Cardiothorac Surg. 2012;41:1083-7.

15. Padilla Alarcón J, Peñalver Cuesta JC. Experience with lung resection in a fasttrack surgery program. Arch Bronconeumol. 2013;49:89-93.

16. Muehling BM, Halter GL, Schelzig H, Meirerhenrich R, Steffen P, SunderPlassman L, et al. Reduction of postoperative pulmonary complications after lung surgery using fast track clinical pathway. Eur J Cardiothorac Surg. 2008; $34: 174-80$.

17. Das-Neves-Pereira JC. Fast-track rehabilitation for lung cancer lobectomy: a five-year experience. Eur J Cardiothorac Surg. 2009;36:383-92.

18. Madani A, Fiore JF, Wang Y, Bejjani J, Sivakumaran L, Mata J, et al. An enhanced recovery pathway reduces duration of stay and complications after open pulmonary lobectomy. Surgery. 2015;158:899-910.

19. Scarci M, Solli P, Bedetti B. Enhanced recovery pathway for thoracic surgery in the UK. J Thorac Dis. 2016;8(Suppl 1):S78-83.

20. Giménez-Milà M, Klein AA, Martinez G. Design and implementation of an enhanced recovery program in thoracic surgery. J Thorac Dis. 2016; 8(Suppl 1):S37-45.

21. Elm von E, Altman DG, Egger M, Pocock SJ, Gøtzsche PC, Vandenbroucke JP. STROBE Initiative. The Strengthening the Reporting of Observational Studies in Epidemiology (STROBE) statement: guideline for reporting observational studies. J Clin Epidemiol. 2008;61:344-9.

22. Gianotti L, Biffi R, Sandini M, Marrelli D, Vignali A, Caccialanza R, et al. Preoperative Oral Carbohydrate Load Versus Placebo in Major Elective Abdominal Surgery (PROCY). Ann Surg. June 2, 2017 [Epub ahead of print].

23. Yeung JH, Gates S, Naidu BV, Wilson MJ, Gao Smith F. Paravertebral block versus thoracic epidural for patients undergoing thoracotomy. Cochrane Database Syst Rev. 2016;2:CD009121.

24. Baidya DK, Khanna P, Maitra S. Analgesic efficacy and safety of thoracic paravertebral and epidural analgesia for thoracic surgery: a systematic review and meta-analysis. Interact Cardiovasc Thorac Surg. 2014;18:626-35.

25. Cerfolio RJ, Bryant AS. Results of a prospective algorithm to remove chest tubes after pulmonary resection with high output. J Thorac Cardiovasc Surg. 2008; 135:269-73.

26. Bjerregaard LS, Jensen K, Petersen RH, Hansen HJ. Early chest tube removal after video-assisted thoracic surgery lobectomy with serous fluid production up to 500 ml/day. Eur J Cardiothorac Surg. 2014;45:241-6.

27. Gustafsson UO, Hausel J, Thorell A, Ljungqvist O, Soop M, Nygren J. Adherence to the enhanced recovery after surgery protocol and outcomes after colorectal cancer surgery. Arch Surg. 2011;146:571-7.

28. ERAS Compliance Group. The impact of enhanced recovery protocol compliance on elective colorectal cancer resection: results from an international registry. Ann Surg. 2015;261:1153-9.

29. Puri V, Patel AP, Crabtree TD, Bell JM, Broderick SR, Kreisel D, et al. Unexpected readmission after lung cancer surgery: a benign event? J Thorac Cardiovasc Surg. 2015;150:1496-504.

30. Zhuang CL, Huang DD, Chen FF, Zhou CJ, Zheng BS, Chen BC, et al. Laparoscopic versus open colorectal surgery within enhanced recovery after surgery programs: a systematic review and meta-analysis of randomized controlled trials. Surg Endosc. 2015;29:2091-100.

31. Bendixen M, Jørgensen OD, Kronberg C, Andersen C, Licht PB. Postoperative pain and quality of life after lobectomy via video-assisted thoracoscopic surgery or anterolateral thoracotomy for early stage lung cancer: a randomised controlled trial. Lancet Oncol. 2016;17:836-44.

32. Falcoz P-E, Puyraveau M, Thomas P-A, Decaluwe H, Hürtgen M, Petersen RH, et al. Video-assisted thoracoscopic surgery versus open lobectomy for primary non-small-cell lung cancer: a propensity-matched analysis of outcome from the European Society of Thoracic Surgeon database. Eur J Cardiothoracic Surg. 2016;49:602-9.

33. Agostini PJ, Naidu B, Rajesh P, Steyn R, Bishay E, Kalkat M, et al. Potentially modifiable factors contribute to limitation in physical activity following thoracotomy and lung resection: a prospective observational study. J Cardiothorac Surg. 2014;9:128.

34. Caastelino T, Fiore JF, Niculiseanu P, Landry T, Augustin B, Feldman LS. The effect of early mobilization protocols on postoperative outcomes following abdominal and thoracic surgery: a systematic review. Surgery. 2016;159: 991-1003.

35. Pompili C, Detterbeck F, Papagiannopoulos K, Sihoe A, Vachlas K, Maxfield MW, et al. Multicenter international randomized comparison of 
objective and subjective outcomes between electronic and traditional chest drainage systems. Ann Thorac Surg. 2014;98:490-6.

36. Fiore JF, Bejjani J, Conrad K, Niculiseanu P, Landry T, Lee L, et al. Systematic review of the influence of enhanced recovery pathways in elective lung resection. J Thorac Cardiovasc Surg. 2016;151:708-15.

Key Words: Enhanced Recovery After Surgery (ERAS), thoracic surgery, lung cancer, Enhanced Recovery Program (ERP), fast-track surgery

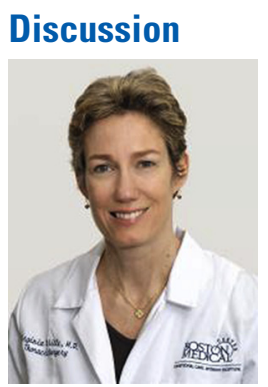

Dr V. Litle (Boston, Mass). ERAS is a new topic for thoracic surgeons, as you pointed out, and I have done an informal poll over the past few days inquiring of our thoracic colleagues, how many are using it, rare, how many are going to be using it, a handful, and then I got several people asking me what ERAS even was. So thank you for bringing this into our field.

You didn't address it here but you did address it in the article, and one of the logical questions would be why didn't you do a randomized trial for this, because if you are really trying to assess implementation of your program, I think it would give you a better idea about how effective the program can be. So maybe you can explain it for the rest of the audience since I got to read about it.

Dr Rogers (Plymouth, United Kingdom). Although not mentioned in this presentation, it was not possible to randomize patients to ERAS or standardized care for this trial because the provision of ERAS required numerous intuitional changes at Bristol Royal Infirmary. All health care professionals involved in the care of thoracic surgery patients required education about the goals and implementation of ERAS protocols. This was particularly important for the ward nursing staff managing the chest drains postoperatively and instigating patient mobilization, but was also true for physiotherapists and junior doctors providing oncall thoracic surgery cover. The implementation of ERAS also required significant developments in the hospital's infrastructure to provide the facilities for one-stop preoperative clinics and a day-of-surgery admission ward.

Dr Litle. How can you incorporate an approach into an ERAS program? I would think that would be a completely separate question about how VATS affected your morbidity. Is there a precedent in the other specialties for incorporating a surgical approach, because obviously you are not going to be able to get everyone to do a VATS.

Dr Rogers. We feel this work shows that an ERAS pathway is beneficial for patients undergoing primary lung cancer resection surgery regardless of approach and therefore feel these protocols can be rolled out in any center wishing to develop an ERAS program. That said, Bristol Royal Infirmary is one of the leading UK centers for VATS and we feel this approach is complimentary, reducing length of stay if not morbidity. These findings are consistent with the evidence base for a laparoscopic approach in colorectal surgery.

Dr Litle. Ideally when you are in a quality improvement program, you want to try to make it as standardized as possible so it could be universally applied, because then your data will be more helpful for others. Also, you identified the importance of early mobilization, but I noted that as high as $50 \%$ of your patients failed to attain early mobilization.

Dr Rogers. You are correct that $50.4 \%$ of patients failed to achieve the satisfactory mobilization within 24 hours of their operation. This was, however, based on very stringent parameters including sitting out of bed for 2 hours the evening after surgery if appropriate and completion of three 60 meter walks around the ward alongside a cumulative 8-hour period sitting out of bed on the first postoperative day. Achieving this was further complicated by the demands this intensive regime placed on nursing staff who are also responsible for patients under a number of different surgical specialities. This is, however, one aspect we are working toward improving.

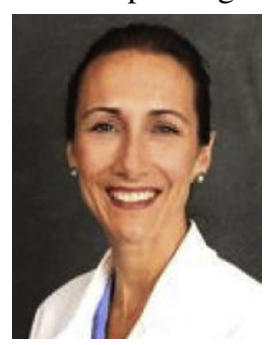

Dr M. B. Marshall (Washington, DC). A lot of us have started to do this without a formalized protocol, so I am curious as to why you did not include nonnarcotic analgesia and intercostal blocks instead of epidurals, which are more troublesome?

Dr Rogers. No patients received epidurals; instead, all patients received a standardized multimodal analgesic strategy that included nonnarcotic analgesia. In addition, patients received paravertebral catheters and, in most cases, patient-controlled analgesia in the form of morphine pump, although we feel this may be unnecessary.

Dr Marshall. Did you have a problem with lack of Foleys in your older male population?

\section{Dr Rogers. No.}

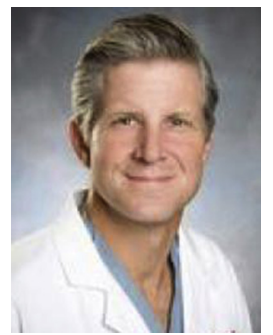

Dr S. Swanson (Boston, Mass). Beyond just getting people up early, is there any other value to this ERAS that you could point out, and what is the PONV, what is the PONV protocol that you use?

Dr Rogers. We feel this study shows that it is not one individual aspect of the ERAS pathway that is beneficial but the aggregation of all of these protocols collectively that results in the reduced morbidity associated with primary lung cancer resection. Although, you are correct that early mobilization 
appears to affect this to a much greater extent. PONV refers to postoperative nausea and vomiting and has a multifactorial etiology. We instituted both nonpharmacologic techniques including preoperative carbohydrate drink and avoidance of crystalloid overload and pharmacologic techniques including intraoperative avoidance of opiates and steroids beside a dose of dexamethasone on induction, intraoperative ondansetron, and regular metoclopramide postoperatively.

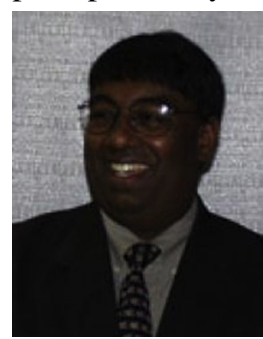

Dr H. Fernando (Falls Church, Va). You mentioned that you don't use a Foley or minimal fluids. How do you manage fluids in the postoperative period, and without a Foley, have you had any issues with a bump in creatinine or renal failure?

Dr Rogers. That is a fair comment and I must say that, if we are concerned with regards to an individual's kidney function preoperatively then some, although a very small minority may have a catheter. In most circumstances, however, this has been limited to those individuals undergoing a pneumonectomy. The Foley catheter will then be removed at the earliest opportunity, ideally within 24 hours. Fluid management was led by the anesthesiologists with an individualized fluid management plan, aiming for euvolemia. This is helped by ensuring patients are as close to euvolemia preoperatively as possible by reducing the time a patient is "nil by mouth" preoperatively to no longer than 2 hours and by facilitating a return to oral intake at the earliest opportunity, often with the patient's first few sips being taken in recovery.

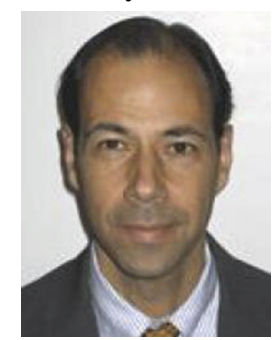

Dr T. D'Amico (Durham, NC). You did not include carbohydrate loading the night before or the 2 days before? Dr Rogers. Patients now receive carbohydrate drinks the evening before surgery, and 6 and 2 hours before surgery. Although during the study period patients only received the carbohydrate drink 2 hours preoperatively.

Dr D'Amico. There is an article in press in the Journal comparing a standard VATS protocol, which has most of these, with the ERAS protocol, which includes the ERAS specific with no difference in outcome. So it does depend on what your baseline care is, but I think your results are interesting.

Dr Rogers. Thank you. I do think we present here strong evidence for an ERAS pathway for all patients undergoing lung cancer surgery, regardless of approach. 